\title{
Optimal Intermittent Dose Schedules for Chemotherapy Using Genetic Algorithm
}

\author{
Nadia Alam ${ }^{\mathrm{a}}$, Munira Sultana ${ }^{\mathrm{a}}$, M.S. Alamª, M A Al-Mamun ${ }^{\mathrm{b}}, \mathrm{M}$ \\ A Hossain ${ }^{\mathrm{b}}$ \\ ${ }^{a}$ Department of Applied Physics, Electronics and Communication Engineering, University of Dhaka, Dhaka-1000, \\ Bangladesh \\ ${ }^{\mathrm{b}}$ Computational Intelligence Group, Faculty of Engineering and Environment, University of Northumbria at \\ Newcastle, UK
}

KEYWORD

Drug Scheduling

Cancer Chemotherapy

Mathematical Model

Optimization

Genetic Algorithm

\begin{abstract}
In this paper, a design method for optimal cancer chemotherapy schedules via genetic algorithm $(G A)$ is presented. The design targets the key objective of chemotherapy to minimize the size of cancer tumor after a predefined time with keeping toxic side effects in limit. This is a difficult target to achieve using conventional clinical methods due to poor therapeutic indices of existing anti-cancer drugs. Moreover, there are clinical limitations in treatment administration to maintain continuous treatment. Besides, carefully decided rest periods are recommended to for patient's comfort. Three intermittent drug scheduling schemes are presented in this paper where GA is used to optimize the dose quantities and timings by satisfying several treatment constraints. All three schemes are found to be effective in total elimination of cancer tumor after an agreed treatment length. The number of cancer cells is found zero at the end of the treatment for all three cases with tolerable toxicity. Finally, two of the schemes, "Fixed interval variable dose (FIVD) and "Periodic dose" that are periodic in characteristic have been emphasized due to their additional simplicity in administration along with friendliness to patients. responses to the designed treatment schedules. Therefore the proposed design method is capable of planning effective, simple, patient friendly and acceptable chemotherapy schedules.
\end{abstract}

\section{Introduction}

Cancer is a class of diseases characterized by an imbalance in the mechanisms of cellular proliferation and apoptosis leading to a solid mass of cells known as a tumor [SLINGERLAND, 1998]. There are four major approaches to cancer treatment: surgery and radiotherapy as local treatments, chemotherapy and the use of biological agents (such as hormones, antibodies and growth factors). However, death is mostly due to spread of the primary tumor to one or more other sites in the body (by a process called metastasis), which makes surgical intervention impracticable [THURSTON, 2006]. Hence, chemotherapy is often applied alone, or in combination with the above methods, as it is the primary method of non-site-specific treatment and distant metastases require a systemic treatment [CLARE, 2000].

Most existing anti-cancer agents act by killing cells that divide fast, one of the main properties of cancer cells. However, cells of the bone marrow, digestive tract and hair follicles also divide fast and therefore harmed by these agents [THURSTON, 2006]. As a result, a regular pattern of side effects is tied to chemotherapy. The common side-effects are myelosuppression (decreased production of blood cells, hence also immunosuppression), mucostisitis (inflamemation of the lining of the gastrointestinal (GI) tract), and alopecia (hair loss). The consistent nature of toxicity limits the dosing in practice. Chemotherapy treatment schedule, defined as dose amount and frequency is needed to be conveniently chosen to lessen the number of cancer cells after a number of 
fixed treatment cycles with acceptable/minimum toxic side effects.

This paper aims in dose designs that take care of some clinical limitations in maintaining continuous treatment and patient's comfort. Treatment methods like intravenous infusion require direct observation of doctors or clinicians and must have occurrence accordingly to the working schedules of the medical professionals. Also if drug administration times are irregular they may lead patients to forget to take their medication or visiting the hospital for treatment [URQUHART et al., 1998]. In addition, to keep toxicity in check, there must be sufficient resting periods. Discontinuous cycles of treatment were suggested by clinicians and in literature to allow periods of recovery of host bone marrow, GI tract, and immune function, expecting that recovery of the tumor cell population would be slower than that of the damaged normal tissues [KAUFMAN et al., 2001; HARROLD, 2005]. This approach let retreatment with high therapeutic doses as frequently as possible. Here we propose three different patterns of dose schedules giving emphasis on the discrete nature in dose administration times. All three cases are planned in such a way that they need to be scheduled only on two subsequent days of a week (Monday and Tuesday for example) that can be selected conveniently. A patient needs to visit hospital for two continuous days on their scheduled weeks only. Rest periods between sessions' are kept to maintain the toxicity in limit. Among the three, the first case is a discrete dosing scheme with arbitrary rest periods. The next two cases offer improved simplicity, regularity and convenience in clinical application along with effectiveness.

Last two decades, drug scheduling of cancer has been approached as both single objective and multi-objective optimization problem. Evolutionary algorithms have been widely engaged to search optimal chemotherapy drug schedule. One of the first mathematical attempts to optimize cancer chemotherapy was by Swan [SWAN, 1986]. Most commonly, logistic growth model was considered for macroscopic tumor proliferation and chemotherapy effects were regarded as bilinear (proportional to drug concentration and tumor size). Martin et al.studied intravenously applieded drug with Gompertzian tumor growth and first-order elimination kinetics of dose effect [MARTIN et al. 1990]. In contrast to the work by Swan, the drug here was considered to be administered weekly - a more applicable treatment methodology based on conventional clinical practice. The single objective was to minimize the final tumor volume without violating toxicity constraints and the optimal amount of drug to administer on a weekly basis over one year treatment was searched. This is a area that was eventually extended by Martin into a book with Teo in [MARTIN et al., 1994]. McCall et al. [MCCALL et al., 1999 [MCCALL et al., 2008] designed chemotherapy drug scheduling using GA nd also prolonged their work with particle swarm optimization (PSO) [MCCALL et al., 2004]. In these works, tumor eradication was used as the objective function and other important treatment requirements, such as maximum drug doses, maximum cumulative drug doses, maximum allowable size of the tumor and toxic side effects were used as constraints for the optimization process that result an effective drug scheduling at the end. The same authors extended their work in multiobjective optimization of cancer chemotherapy scheduling via multi-objective GA (MOGA) [MCCALL et al., 2004]. Algoul et al. applied MOGA [ALGOUL et al., 2010; ALGOUL et al. 2011] and MOPSO [ALAM et al., 2013] in their works for dose designing in closed-loop control to minimize the conflicting objectives of cell killing and toxic side-effect and continuous dose schedules were developed. Though multiobjective evolutionary algorithms balance different conflicting treatment objectives, single-objective optimization has been also providing efficient dose schedules with very satisfactory result. [ALAM et al., 2013a] presented a work where three drug scheduling schemes are proposed where GA is used to optimize the doses and schedules by satisfying several treatment constraints. Therefore, highlighting on clinical relevance and patient's ease, this paper presents a design method of optimal cancer chemotherapy schedules where genetic algorithm (GA) is used to optimize drug doses and intervals by minimizing the treatment objective of minimizing cancer cell number and without violation of four key constraints: i) 
maximum drug concentration, ii) maximum toxicity, iii) maximum cumulative drug concentration and iv) maximum allowable tumor size. Here we worked using the model developed by Martin et al. [MARTIN et al., 1994] that accounts for an intravenously administered drug with first-order elimination acting on a tumor going through Gompertzian proliferation. Apart from the statistical modeling of tumour growth, mathematical models of tumour growth in tissue [ALMAMUN et al., 2012], cellular and sub-cellular level [AL-MAMUN et al., 2013; KAZMI et al., 2012a; KAZMI et al., 2012b] give the idea about the tumour dynamics in a comprehensive way. In most of cases, the Gompertz model of tumour growth has been taken for the drug scheduling problem, but in [KAZMI et al., 2012a] considered the cellular growth model drug transport model where hypoxic area has been divided into five regions to show the drug bindings in the protein networks.

\section{Mathematical model}

Here we are considering a system, originally studied by Martin et al. [MARTIN et al., 1994], that describes a tumor proliferating in Gompertzian fashion and an intravenous application of drug. The pharmacokinetics (PK) of the drug is illustrated by the DE given below.

$$
\dot{C}(t)=D(t)-C(t)
$$

where the plasma drug concentration, $C(t)$, increases with intravenous infusions of the drug, $D(t)$, and decreases according to first-order elimination kinetics at a rate $\lambda$. Equation 1 represents the increase in drug concentration at the tumor site. As a result of intravenous delivery of the drug, an immediate mixing of the drug with the plasma is assumed. $\lambda$ is related to the half-life of the drug as: $\ln (2) / \lambda$. The change in the number of cancer cells is described by another Equation.

$$
\begin{aligned}
\dot{N}(t)=\frac{1}{\tau_{g}} & {\left[\frac{\ln \left(\frac{\rho_{g}}{N_{0}}\right)}{\ln \left(\frac{\rho_{g}}{2 N_{0}}\right)}\right] N(t) \ln \left(\frac{\rho_{g}}{N(t)}\right) } \\
& -k_{e f f} C_{e f f}(t) N(t)
\end{aligned}
$$

According to Equation 2, the cancerous cells proliferate in a Gompertzian fashion. As tumor size increases, the tumor growth slows and the mass approaches a plateau population [NORTON, 1988]. This type of growth is normally described using the Gompertz Equation [MARTIN et al., 1994; HARROLD, 2005] for tumor growth. The $1^{\text {st }}$ term in the right side of Equation 2 is the Gompertzian proliferation term, where $\varrho_{g}$ is the asymptotic plateau population, $N_{0}$ is the initial number of tumor cells, and $\tau_{g}$ is the doubling time of the tumor during exponential growth [MARTIN et $a l .$, 1994]. The therapeutic effect of the drug on the tumor is then represented mathematically by adding a bilinear kill term to the tumor growth Equation which is the $2^{\text {nd }}$ term on right of Equation 2. The added nonlinear term is proportional to both the current size of the tumor, $N(t)$, with constant of proportionality $k_{\text {eff }}$ and the effective drug plasma concentration, $C_{\text {eff }}(t)$ [MARTIN et al., 1994]. The parameter $k_{\text {eff }}$ is the proportion of tumor cells killed per unit time per unit drug concentration and termed as fractional kill term. $C_{\text {eff }}(t)$ is the drug concentration above the minimum therapeutic concentration, $C_{t h}$, as given in Equation 3 . Equation 4 is a Heaviside step function that implies drugs may not become effective until a therapeutic plasma concentration is reached $\left(C_{t h}\right)$. Once $C_{t h}$ has been reached, the effective drug concentration $\left(C_{e f f}\right)$ is that concentration above $C_{t h}$ as expressed in Equation 3.

$$
\begin{aligned}
& C_{e f f}(t)=\left(C(t)-C_{t h}\right) H\left(C(t)-C_{t h}\right) \\
& H\left(C(t)-C_{t h}\right)= \begin{cases}1, & \text { if } C(t) \geq C_{t h} \\
0, & \text { if } C(t)<C_{t h}\end{cases}
\end{aligned}
$$

The initial drug concentration and number of cancer cells are given by $C_{0}$ and $N_{0}$, respectively. 


$$
\begin{aligned}
& C(0)=C_{0}=0 \\
& N(0)=N_{0}
\end{aligned}
$$

Finally, the toxicity is modeled as follows [ALGOUL et al., 2010].

$$
\dot{T}(t)=C(t)-\eta T(t)
$$

Equation 7 gives the toxicity level in body after infusion of drugs where $\eta$ is a constant. It gives the toxicity level for the drug concentration in plasma at a time. The parameters considered for this case study [MARTIN et al., 1994; ALGOUL et al., 2010] are provided in Table (1). In next section we are going to discuss our design objectives and applied constraints on the model considered to achieve optimal treatment schedule.

\begin{tabular}{llll}
\hline PARAMETER & DESCRIPTION & VALUE & UNIT \\
\hline$\tau_{g}$ & First doubling time of the tumor during exponential growth & 150 & Days \\
$\varrho_{g}$ & Plateau population of cancer cells without treatment & $10^{12}$ & Cells \\
$N_{0}$ & Initial cancer cell population & $10^{10}$ & Cells \\
$k_{e f f}$ & Fractional cell kill term for a highly effective drug & $2.7 \times 10^{-2}$ & $1 /$ days.[D] \\
$\lambda$ & Decrease in concentration of drug per unit time & 0.27 & $1 /$ days \\
$\eta$ & Toxicity rate constant & 0.4 & $1 /$ days \\
$C_{t h}$ & Threshold drug concentration in plasma & 10 & [D] \\
$C_{\max }$ & Maximum tolerable drug concentration & 50 & [D] \\
$C_{c u m}$ & Maximum tolerable total drug exposure in plasma & $4.1 \times 10^{3}$ & [D].days \\
$T_{\max }$ & Maximum tolerable toxicity & 100 & [D] \\
$t_{f}$ & Duration of treatment schedule & 364 & Days \\
\hline \multicolumn{4}{c}{ Table. 1. Parameters Used in the model [MARTIN et al., 1994; ALGOUL et al,. 2010]. Here [D] are the units of drug }
\end{tabular}

\section{Design constraints and objective}

Here we considered three types of toxicity constraints and an efficacy constraint.

- Maximum Drug Concentration: Acute toxicity is reached when the drug plasma concentration exceeds some maximum, $C_{\max }$ [MARTIN et al. 1994; HARROLD, 2005]. This constraint is given by

$$
C(t) \leq C_{\max } ; \forall t \in\left[0, t_{f}\right]
$$

- Maximum Toxicity: Along with the acute drug concentration, a constraint on the measurement of toxicity [ALGOUL et al., 2010] is used as follows.

$$
T(t) \leq T_{\max } ; \forall t \in\left[0, t_{f}\right]
$$

The toxicity $T(t)$ at any time must not exceed $T_{\max }$

- Maximum Cumulative Drug Concentration: Total exposure of drugs in plasma can be calculated by integrating drug plasma concentration over the treatment interval and must not exceed a value $C_{\text {cum }}$ [MARTIN et al., 1994; HARROLD, 2005].

$$
\int_{0}^{t_{f}} C(t) d t \leq C_{\text {cum }}
$$

- Efficacy constraint: Since it was undesirable for the tumor burden to increase, the number of cancer cells was not allowed to increase to a number larger than the initial condition, $\mathrm{N}_{0}$ [HARROLD, 2005]. Therefore efficacy constraint can be expressed as below.

$$
N(t) \leq N_{0} ; \forall t \in\left[0, t_{f}\right]
$$


The values of $C_{\max }, T_{\max }$ and $C_{\text {cum }}$ are included in Table (1). Here we have employed the optimal control problem considered by Martin et al. [MARTIN et al., 1994] to minimize cancer cell no. at a final time. It can be stated as:

$$
\begin{gathered}
M I N_{D(t)} N\left(t_{f}\right) \\
\text { s.t. }(1-11)
\end{gathered}
$$

The objective here is to minimize the tumor volume at a final time while satisfying the dynamic constraints (equation 1-7), toxicity constraints (inequalities 8-10), and the efficacy constraint (11). In other words, we have to design a chemotherapy schedule for 364 days to minimize the final number of cancer cell. The drug concentration should range between 10 and 50 and cumulative drug concentration should be lower than a value $4.1 \times 10^{3}$. Finally, the cancer cell number should never exceed $10^{12}$.

\section{Genetic algorithm}

Genetic algorithm (GA) is a stochastic global search procedure that replicates the metaphor of natural biological evolution introduced by Holland [HOLLAND, 1975]. Fig. 1 shows a schematic of GA applied to a single objective minimization problem. The element processed is a string formed by concatenating sub-strings, each of which is a numeric coding of a control variable. Each string represents a solution (or an individual) in the search space. A set of solutions/individuals used in one generation of an EA is called a population [DEB, 2008].

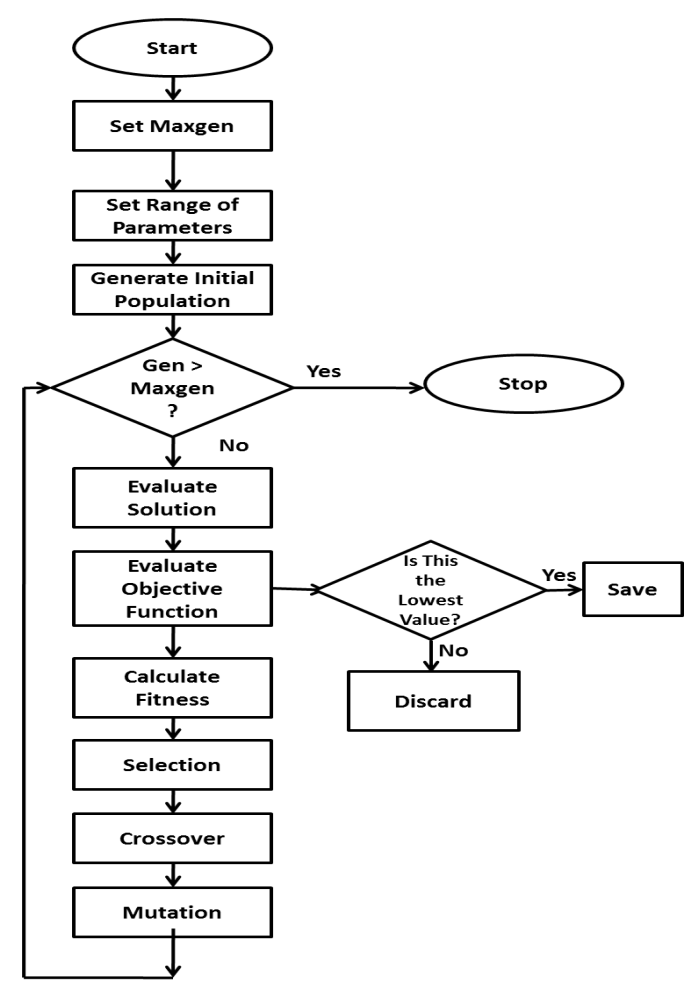

Fig. 1. A flowchart of genetic algorithm optimization

GA uses three main operators - selection, crossover and mutation - on a population of solutions at every generation. Performance of each solution is assessed through an objective function determined by the problem and used in selection operation to guide the search towards the best solution. New solutions formed by a combined effect of crossover and mutation operators [DEB, 2008]. Crossover can cause interchange of the traits between any two solutions (chromosomes) via random decision in the mating pool and provides a way to produce the desired qualities. Although selection and crossover provide most of the power skills, the area of the solution remains limited. Mutation can operate on a single solution to create a new perturbed solution by random alternation of digits and thus keeps diversity in population. The population generation process is repeated for sufficient number of times and the last generated population is expected to contain a solution that minimizes the objective function. 


\section{Optimal chemotherapy schedule using GA}

Here we present three drug scheduling schemes, all intended for 364 days and express them in terms of decision variable. Then GA is encoded and employed to find optimal dose schedules according to each of the schemes.

\subsection{Variable interval variable dose (VIVD) scheme}

In VIVD scheme, chemotherapy treatment will be administered to patients only first two days of each week depending on decision variable. Fig. 2 shows a schematic of the dose plan. For each week, decision variable is encoded with one bit; ' 1 ' to indicate that a patient will receive treatment on that week and ' 0 ' to indicate rest week, i.e., no drugs will be administered on that week. Giving clinical relevance, same drug doses are administered to patient treatment for first two days of any treatment week and one variable is required for each week. So, two variables are defined for each week; one for dose and one for decision. For a year (364 days $=52$ weeks) long treatment plan, $52 \times 2=104$ variables are required and GA is used find an optimum solution set.

\subsubsection{Encoding GA}

To optimize dose schedule in VIVD scheme, the GA optimization process begins with a randomly generated population of size $50 \times 676$

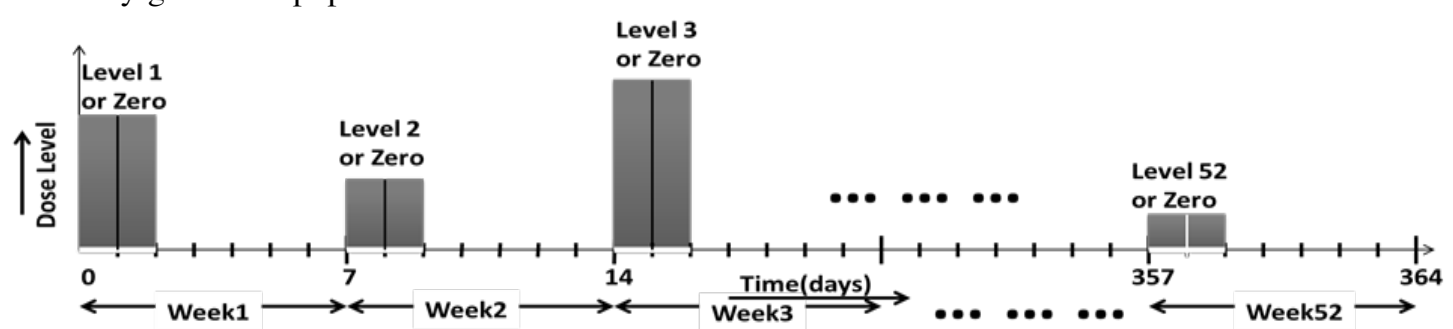

Fig. 2. A schematic of variable interval variable dose (VIVD) scheme

following generations. Once solutions are evaluated, fit solutions are selected through selection process to form the mating pool. Crossover and mutation are applied to obtain the where 50 is the number of individuals and $676(=(52 \times 12)+(52 \times 1))$ is the length of the solution string for 104 control variables. First 52 parameters are encoded as 12 bits binary strings which will define drug doses for each week while the remaining 52 parameters are encoded as 1 bit to define decision variables, i.e., whether treatment will be given to a patient. First 52 binary strings are converted into real numbers within a range of 10 to 50 . Using each individual (solution), a chemotherapy drug schedule is designed for one year as discussed earlier and used as input $D(t)$ to the tumor model stated in Section 2. The model is simulated and several important output parameters: number of cells, drug concentration and toxicity are measured. The number of tumor cells at the end of treatment is used as objective function in GA optimization process.

Before calculating objective function, each solution is checked for constraints. If any of the constraint is not satisfied, that solution is penalized by adding a big penalty value so that it will have less chance to be selected for

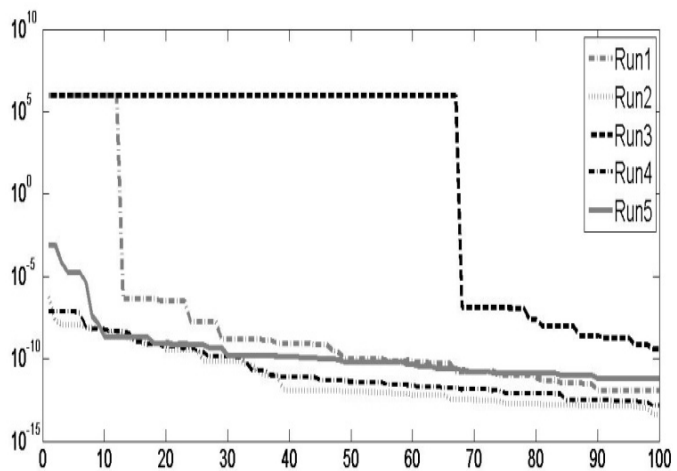

Fig. 3. Convergence of GA for VIVD scheme off-spring population. The crossover rate and mutation rate are set as 0.8 and 0.01 respectively. The maximum number of generations is set to 100 . It is noted that, binary- 
coded GA is preferred and used in this optimization/design procedure because half of the control variable $(=52)$ are binary-type decision variables represented by a single bit. $\mathrm{GA}$ with said parameters is run several times on the model. Fig. 3 shows algorithm convergence in five runs. Table. 2 gives a summary of the performance measures of the dose schedules decided by five different runs.

\begin{tabular}{cllllllll}
\hline Run & \multicolumn{2}{l}{ Drug Dose } & \multicolumn{2}{c}{ Drug Concentration } & Toxicity & No. Cell & $\begin{array}{l}\text { Cell } \\
\text { at end }\end{array}$ \\
\cline { 2 - 6 } & Max & Avg & Max & Avg & Max & Avg & adion \\
\hline 1 & 32 & 10.73 & 49.45 & 11.23 & 83.41 & 27.77 & $1 \times 10^{-12} \approx 0$ & $\approx 100 \%$ \\
2 & 32 & 10.56 & 49.98 & 11.14 & 83.35 & 27.84 & $4 \times 10^{-14} \approx 0$ & $\approx 100 \%$ \\
3 & 32 & 10.67 & 49.58 & 11.22 & 81.37 & 27.94 & $4 \times 10^{-10} \approx 0$ & $\approx 100 \%$ \\
4 & 32 & 10.71 & 49.81 & 11.22 & 84.72 & 27.76 & $1 \times 10^{-13} \approx 0$ & $\approx 100 \%$ \\
5 & 32 & 10.73 & 49.91 & 11.23 & 82.29 & 27.79 & $6 \times 10^{-12} \approx 0$ & $\approx 100 \%$ \\
\hline \multicolumn{7}{c}{ Table. 2. Performance measures for dose schedules obtained by five GA runs with VIVD scheme }
\end{tabular}

\subsubsection{Performance}

The numbers of cancer cell at the end of the treatment (objective function), in response to the dose schedules by all five runs are zero (practically the number of cancer cell cannot be fraction) as can be seen in Table. 2. Moreover, the maximum and average values of delivered drug dose, plasma drug concentration and toxicity for the determined drug schedules in all the five runs, presented in Table. 2 are also very close to one another. All these imply repeatability and consistency of GA optimization process as well as the whole design procedure. Since all five dose schedules more or less show same levels of effectiveness and safety, we here discuss the one decided in case of run-1 only. The optimal treatment schedule for one year in VIVD scheme obtained by GA run-1 is shown in Fig. 4. The tumor cell no., drug concentration and toxicity values in response to the treatment are shown respectively in Fig. 5, Fig.6 and Fig.7.

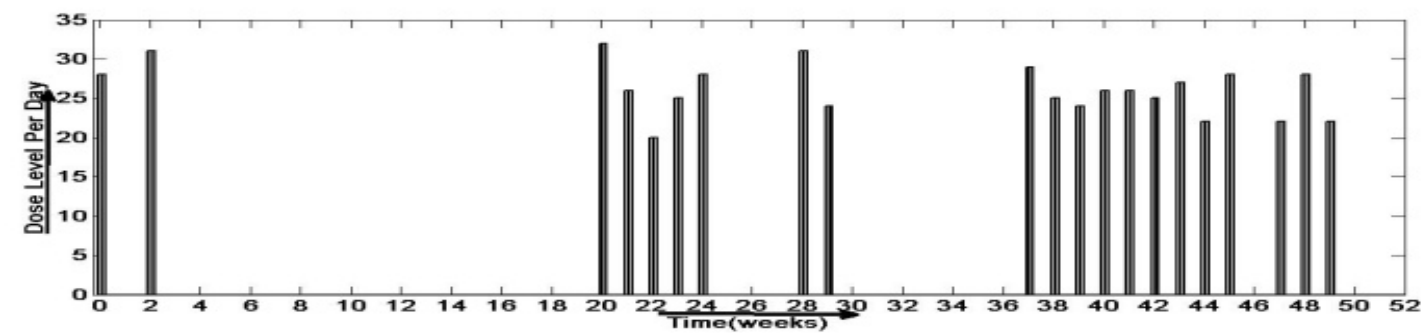

Fig. 4. An optimal treatment schedule for one year (52 weeks) in VIVD scheme

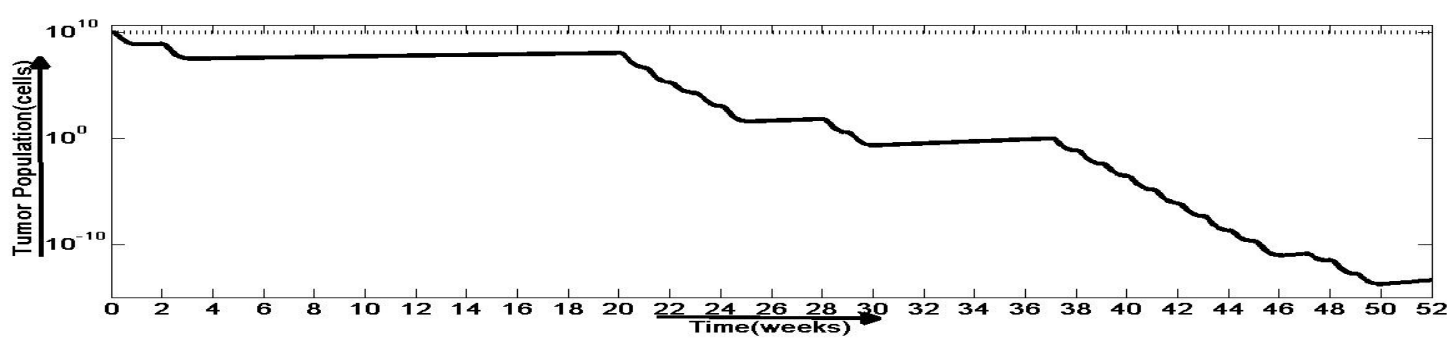

Fig. 5. Tumor cell no. in response to treatment schedule in VIVD scheme 


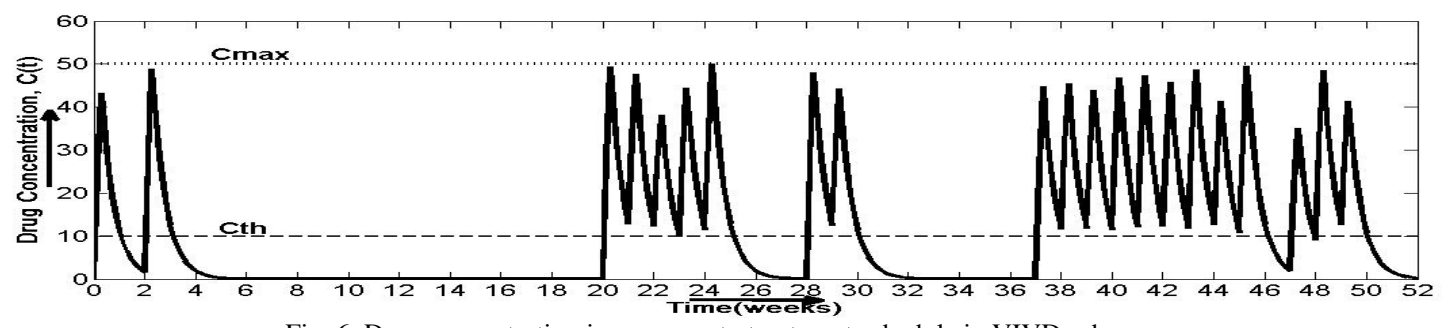

Fig. 6. Drug concentration in response to treatment schedule in VIVD scheme

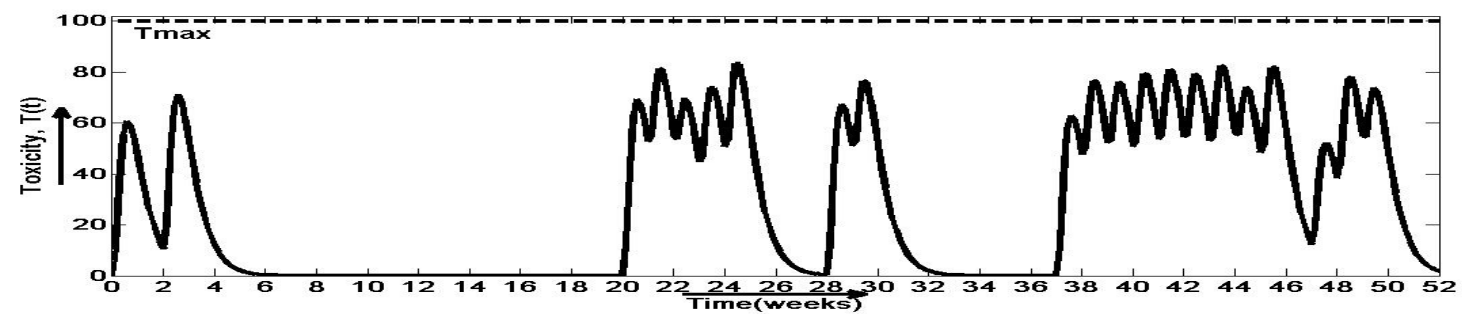

Fig. 7. Toxicity in response to treatment schedule in VIVD scheme

In Fig. 5, the dose level in a particular day is given by the bar height and each bar starting at beginning of a week is continuous for two consecutive steps ( 1 step $=1$ day) indicating that same level of dose is applied for two consecutive days. It is a random scheme where the scheduled weeks are determined by the optimal solutions of 52 decision parameters. It can be easily seen from Fig. 4, weeks to be dosed determined by the decision parameters are week no. $0,2,20,21,22,23,24,28,29,37$, 45, 47, 48 and 49. For these weeks the value of decision parameters are ' 1 '. And for other weeks, the schedule suggests dosing to be skipped and the corresponding decision parameters are ' 0 '. The dose is applied to $1^{\text {st }} 2$ days of each arbitrarily decided week according to the corresponding solution of dose level parameter. The tumor population decreases from the $1^{\text {st }}$ day of treatment and reduces to approximately zero as suggested by Fig. 5 which is a logarithmic plot of number of tumor cell during the treatment period. Fig. 6 and Fig. 7 show drug concentration and toxicity due to dosing which are maintained in limit. The rest periods between sessions help to keep the toxicity in control.

\subsubsection{Remarks}

The treatment plan discussed above is irregular, therefore lacks clinical and logical acceptability in dose administration. As it was able to meet the constraints, we can term the solution as mathematically optimal. But the plan involves too much information to record. Also since the dosing is at random weeks, a patient may forget to visit clinic/hospital for administration and miss a scheduled session. Our next step towards clinical relevance is to propose a regular schedule with lesser parameters and optimize those parameters as before.

\subsection{Fixed interval variable dose (FIVD) scheme}

In FIVD, interval between two successive sessions is fixed during the course of the whole treatment period. Drugs are administered to patients on first two days of every $4^{\text {th }}$ week (e.g. weeks $4,8,16$ etc.) following a rest period of 26 days. For any treatment week, same doses are administered on first two days. So, only one control variable is required to define the dose level of any treatment week. Fig. 8 indicates that treatments are given only in 52/4=13 weeks and so 13 variables (each representing dose level for a week) are required in designing this dose pattern. Targeting clinical relevance and to achieve treatment efficacy, a high dose, called bowl (fixed dose level of 50[D]) is administered at the start of the treatment. 


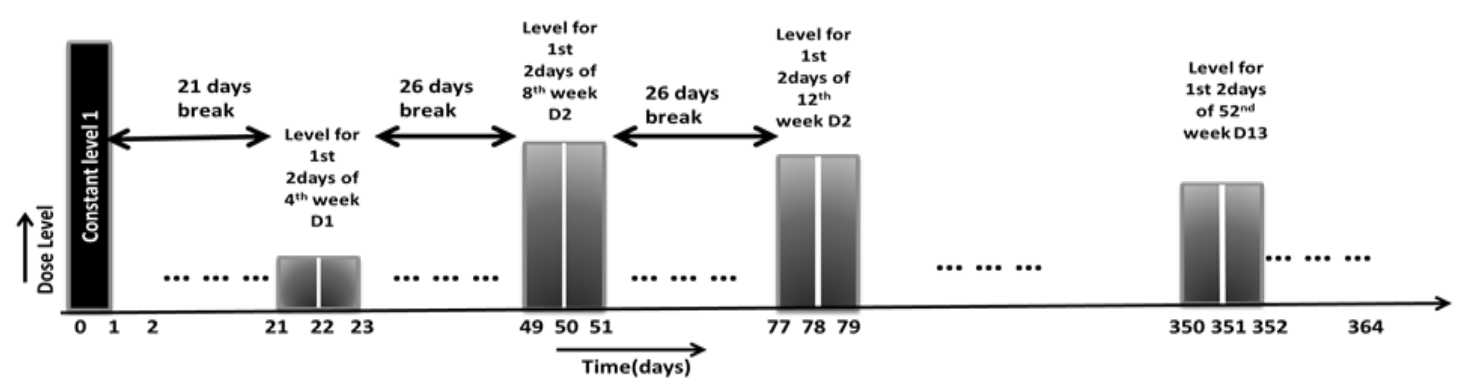

Fig. 8. A schematic of fixed interval variable dose (FIVD) scheme.

\subsubsection{Encoding GA}

The GA encoding is similar to the one for the VIVD pattern. Only difference is the number of control variables is 13 as the dose plan needs. The dose input $D(t)$ is modified accordingly with 13 parameters. In this case the maximum generation is taken to be 50 and the values of other GA parameters are also kept unchanged. GA optimization process is run 3 times on the model for this case. The convergence curves for all runs are shown in Fig. 9. Again each run generates a dose schedule for 364 days according to the pattern that fulfills the declared constraints. Table. 3 gives some performance measures for all three runs.

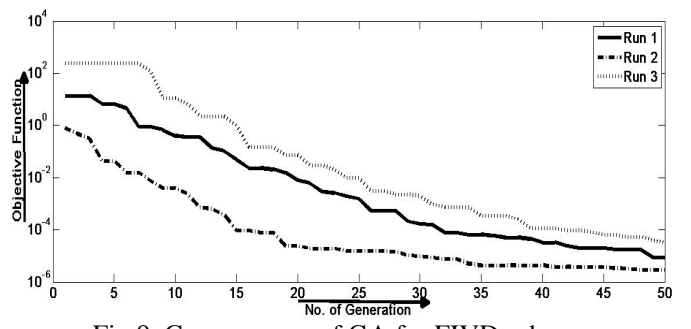

Fig.9. Convergence of GA for FIVD scheme

\begin{tabular}{|c|c|c|c|c|c|c|c|c|}
\hline \multirow[t]{2}{*}{ Run } & \multicolumn{2}{|c|}{ Drug Dose } & \multicolumn{2}{|c|}{ Drug Concentration } & \multicolumn{2}{|l|}{ Toxicity } & \multirow{2}{*}{$\begin{array}{l}\text { No. Cell } \\
\text { at end }\end{array}$} & \multirow{2}{*}{$\begin{array}{l}\text { Cell } \\
\text { Reduction }\end{array}$} \\
\hline & Max & Avg & Max & Avg & $\operatorname{Max}$ & Avg & & \\
\hline 1 & 50 & 32 & 49.5674 & 8.6573 & 69.9071 & 21.3205 & $9 \times 10^{-6} \approx 0$ & $\approx 100 \%$ \\
\hline 2 & 50 & 32.5926 & 49.5674 & 8.8161 & 69.9071 & 21.7071 & $3 \times 10^{-6} \approx 0$ & $\approx 100 \%$ \\
\hline 3 & 50 & 31.4074 & 49.4778 & 8.4914 & 69.525 & 20.8953 & $3 \times 10^{-5} \approx 0$ & $\approx 100 \%$ \\
\hline
\end{tabular}

Table. 3. Performance measures for dose schedules obtained by three GA runs with FIVD scheme

\subsubsection{Performance}

Like the previous scheme, the final numbers of cancer cell for all GA runs with FIVD scheme are zero (see Table. 3). All the average values were taken per day considering the whole treatment period as before except the average drug dose. For calculating the average dose delivered per day, we only considered the scheduled dose days $(13 \times 2+1=27$ days in this case) in place of the total treatment period (364 days). The average values of toxicity and drug concentration are lower than those in previous case (which is desired). Alike the previous case, performance measures for FIVD, recorded and presented in Table. 3 , are also very close to one another. Therefore, the optimal treatment schedule for one year in FIVD scheme attained by GA run-2 is displayed in Fig. 10. The tumor cell no., drug concentration and toxicity values in response to the treatment are displayed respectively in Fig. 11, Fig.12 and Fig.13. 


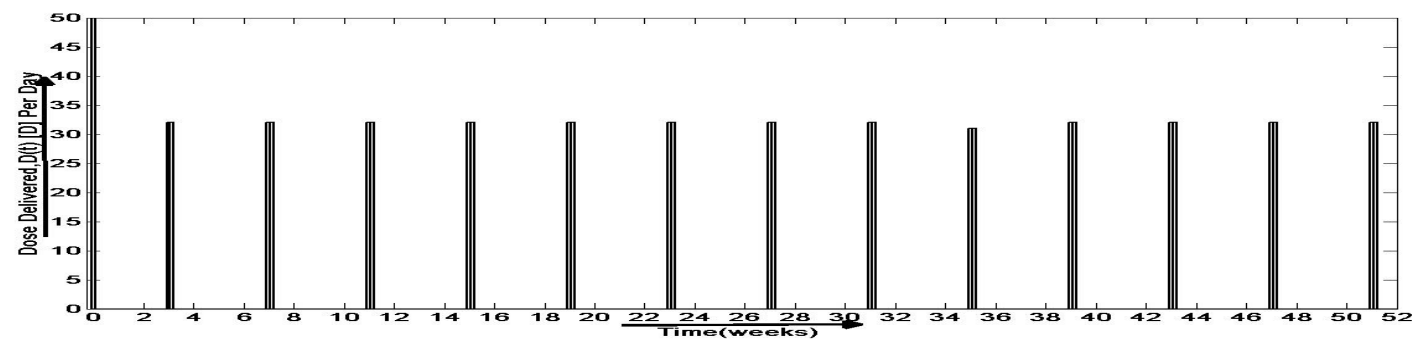

Fig. 10. An optimal treatment schedule for one year (52 weeks) in FIVD scheme

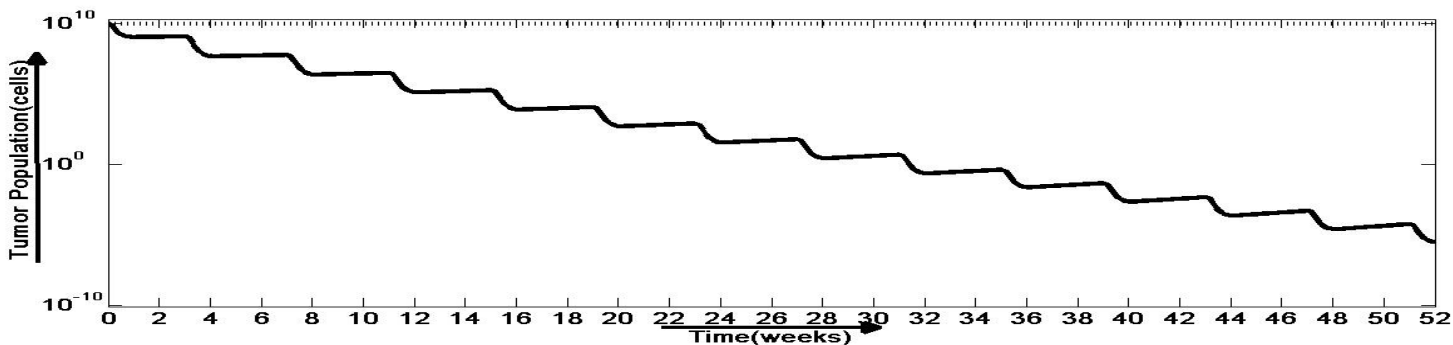

Fig. 11. Tumor cell no. in response to treatment schedule in VIVD scheme

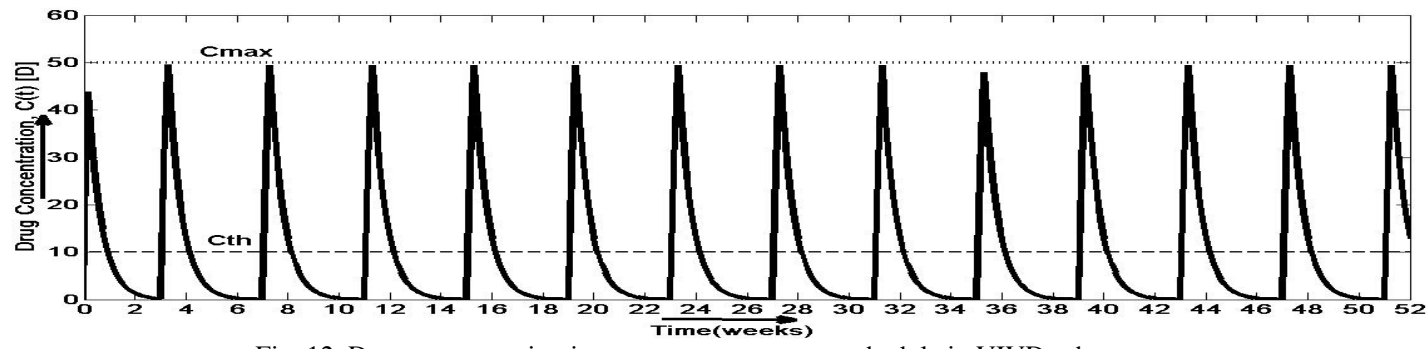

Fig. 12. Drug concentration in response to treatment schedule in VIVD scheme

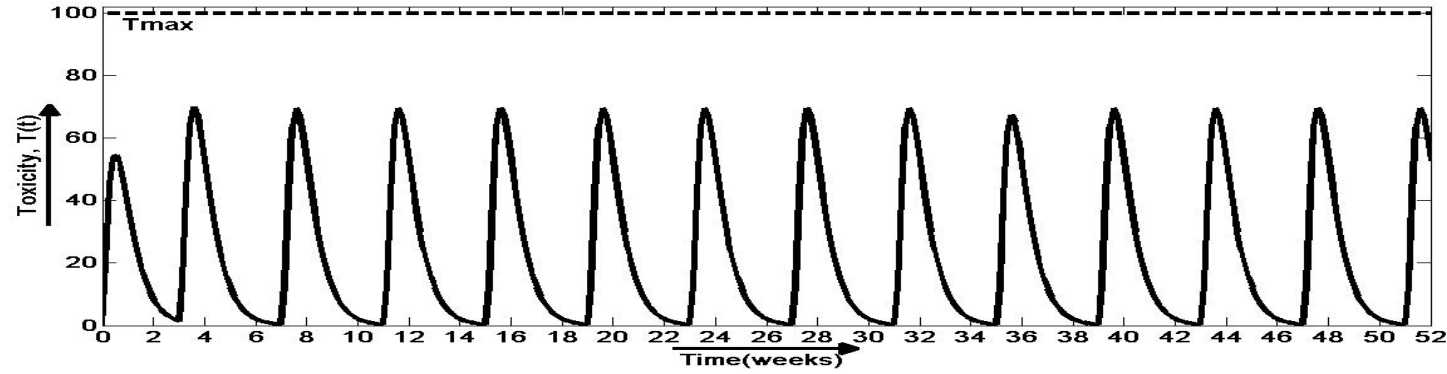

Fig. 13. Toxicity in response to treatment schedule in VIVD scheme

On the starting week (week 0 on Fig. 10), only the $1^{\text {st }}$ day is decided with a constant dose of $50[D]$ in order to fulfill efficacy constraint. For the $4^{\text {th }}$ weeks (denoted by week $3,7,11,15 \ldots$, 51), the weekly levels are found after solving 13 parameters with GA optimization fulfilling the given constraints. This dose scheme looks almost periodic after week 3 as the $4^{\text {th }}$ weekly levels are almost same. Fig. 11 shows how the tumor cell no. reduces gradually to a value $\approx 0$. Drug concentration and toxicity changes as displayed in Fig. 12 and Fig. 13 show a rough periodicity according to the dose plan and their limiting values $\left(C_{\max }\right.$ and $\left.T_{\max }\right)$ are not exceeded. 


\subsubsection{Remarks}

This dose plan leads more towards clinical applicability. Decrease in number of parameters, regularity in treatment schedule, decrease in toxicity and efficacy constraint assurance are its features. Though it is not precisely periodic, from the $4^{\text {th }}$ week a near periodicity is visible. If we consider solution of any of the runs, the weekly dose levels vary from 30 to 32 . So if it is desired the scheme can be easily modified to a periodic one.

\subsection{Periodic dose scheme}

In this scheme, scheduled days and intervals are same as FIVD. However, in any treatment week, different valued drug doses are used on first two days. Having two separate levels is just an additional variation to get a better treatment response. Same doses are periodically administered in subsequent treatment weeks. Fig. 14 shows a schematic of the dose plan. As a result only two control variables are required to design treatment schedules for a year.

\subsubsection{Encoding GA}

The dose input $D(t)$ in this case requires 2 parameters this time which are two dose levels repeated every $4^{\text {th }}$ week. GA parameters are also kept same. GA process with 50 generation is run3 times on the model. The convergence curves for all runs are shown in Fig. 15. Table. 4 presents some performance measures.

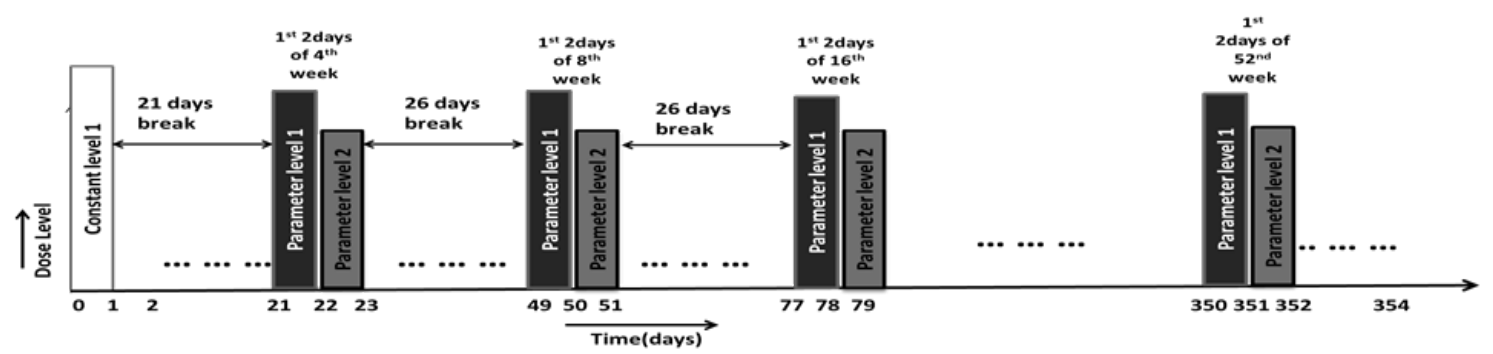

Fig.14. A schematic of periodic dose scheme.

\begin{tabular}{|c|c|c|c|c|c|c|c|c|}
\hline \multirow[t]{2}{*}{ Run } & \multicolumn{2}{|c|}{ Drug Dose } & \multicolumn{2}{|c|}{ Drug Concentration } & \multicolumn{2}{|l|}{ Toxicity } & \multirow{2}{*}{$\begin{array}{l}\text { No. Cell } \\
\text { at end }\end{array}$} & \multirow{2}{*}{$\begin{array}{l}\text { Cell } \\
\text { Reduction }\end{array}$} \\
\hline & Max & Avg & Max & Avg & $\operatorname{Max}$ & Avg & & \\
\hline 1 & 50 & 34.5926 & 49.3403 & 9.3646 & 74.3991 & 23.0717 & $3.7 \times 10^{-8} \approx 0$ & $\approx 100 \%$ \\
\hline 2 & 50 & 34.5926 & 49.3403 & 9.3646 & 74.3991 & 23.0717 & $3.7 \times 10^{-8} \approx 0$ & $\approx 100 \%$ \\
\hline 3 & 50 & 34.5926 & 49.9624 & 9.3631 & 74.3703 & 23.0654 & $4.1 \times 10^{-8} \approx 0$ & $\approx 100 \%$ \\
\hline
\end{tabular}

\subsubsection{Performance}

Likewise the previous cases, final tumor cell numbers are once again $\approx 0$. Averages are calculated similarly as last case. In this case, the average drug doses for all runs are same $34.5926[\mathrm{D}]$. It may be also mentioned that both run-1 and run-2 generate exactly same optimal dose schedule (same parameters values). Also performance of the schedule obtained by run-3 is very close them. The optimal treatment schedule in periodic dose scheme decided by GA run-1/run-2 is displayed in Fig. 15. The tumor cell no., drug concentration and toxicity values in response to the treatment are displayed respectively in Fig. 16, Fig.17 and Fig.18. 


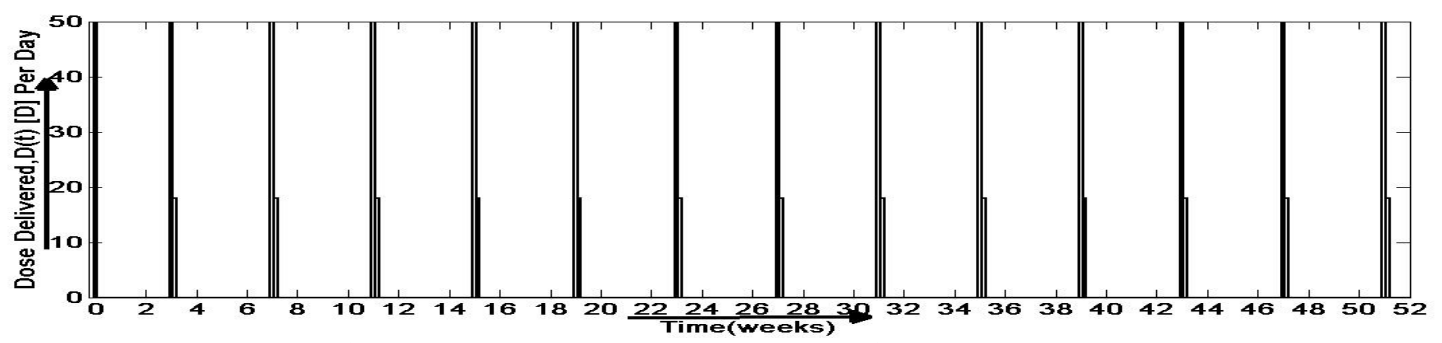

Fig. 15. An optimal treatment schedule for one year (52 weeks) in FIVD scheme

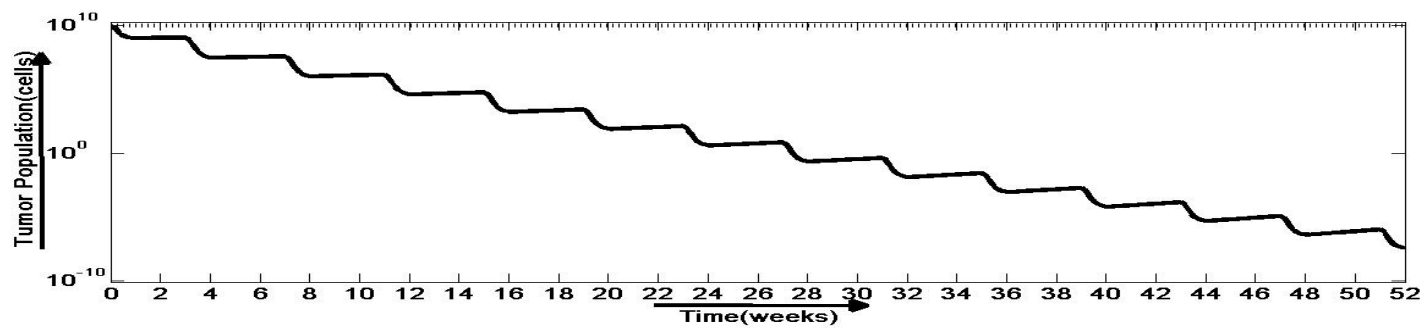

Fig. 16. Tumor cell no. in response to treatment schedule in FIVD scheme

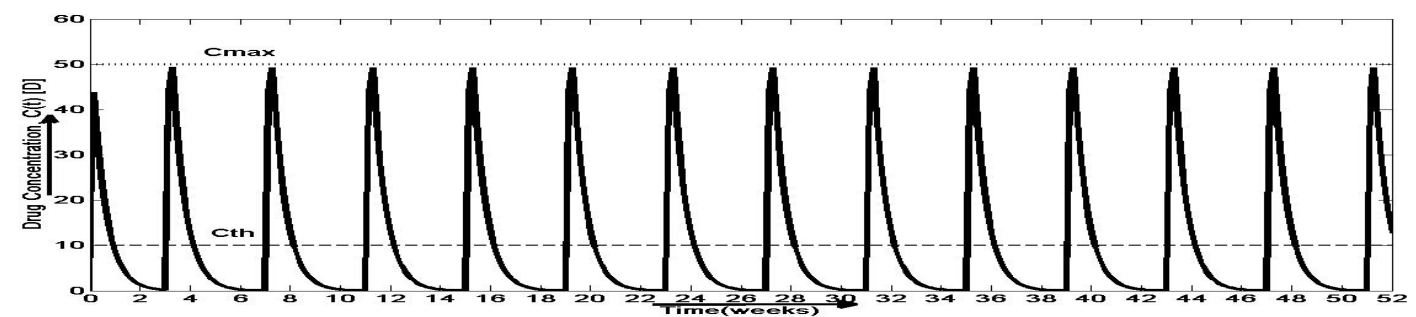

Fig. 17. Drug concentration in response to treatment schedule in FIVD scheme

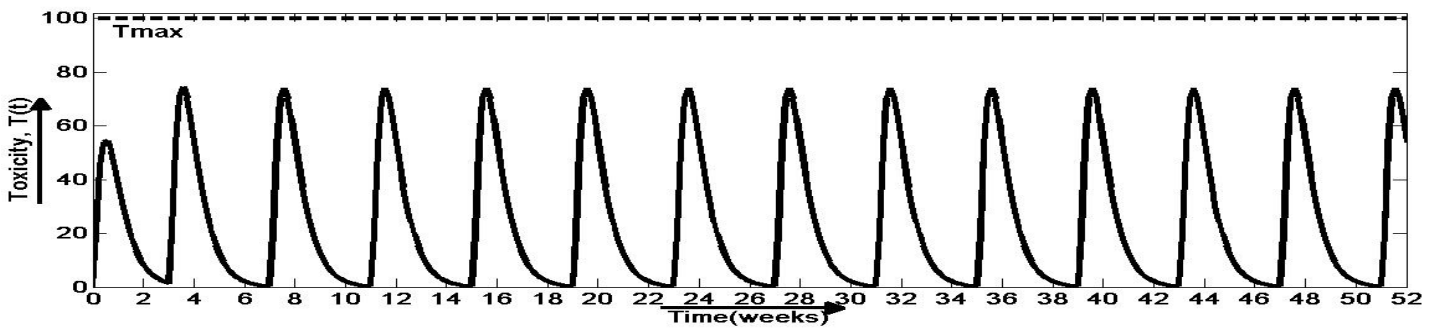

Fig. 18. Toxicity in response to treatment schedule in FIVD scheme

Fig. 15 shows an optimal dose schedule starting with a pre-decided constant dose concentration/mass of 50[D] applied to guarantee efficacy constraint. For every $4^{\text {th }}$ week the dosing can be clearly seen to be periodic. Each $4^{\text {th }}$ week is dosed with $50[\mathrm{D}]$ on the $1^{\text {st }}$ day followed by $18[D]$ on the $2^{\text {nd }}$ day. The remaining days of a week are kept as rest period for the patient to recover from toxic side effects, if occur or tend to occur. Like previous cases, drug concentration and toxicity levels have not surpassed their limiting values $\left(C_{\max }\right.$ and $\left.T_{\max }\right)$.

\subsubsection{Remarks}

This dose plan can be referred as a strictly periodic one from $4^{\text {th }}$ week in terms of repeated discrete levels of doses with constant periodicity. Periodicity is encouraged for clinical implementation. Moreover, it is highly simplified, patient friendly but still effective. 
The optimal solutions in all three dose schemes have led to $100 \%$ elimination of cancer tumors. Furthermore, maximum toxicity levels during the whole period of treatment remain lower than the maximum allowable value as indicated earlier and suggested by other researchers [MARTIN et al., 1994; HARROLD, 2005; ALGOUL et al., 2010]. Also both maximum and cumulative drug concentration are managed in limit. The fulfillment of these three constraints of toxicity is ensured by intermittent treatment. Such treatments are clinically encouraged [HARROLD, 2005; KAUFMAN et al., 2001; ADAMS et al., 2011] for preserving the quality of life of patients along with the cure of disease. Also the resting period must be carefully chosen based on the therapeutic index (ratio of effectiveness and toxicity) [THURSTON, 2006] of the applied drug. It must not be too large to face deterioration of cancer condition and must be small enough for recovery from toxic side effects [KAUFMAN et al. 2001]. To provide this, for both FIVD and periodic dose schemes, a fixed interval of 26 days has been decided. This approach has also let retreatment with high therapeutic doses on scheduled days as suggested by Kaufman et al. [KAUFMAN et al., 2001]. Moreover, this kind of schedule offers to be more time effective and well manageable for medical professionals as compared to continuous treatments with lower doses as in [ALGOUL et al., 2010; ALGOUL et al. 2011].

Among those three dose patterns presented in this work, the first one was planned with flexibility with dosing occurrence. After applying GA optimization, it resulted in an intermittent dosing scheme with variable resting period and named variable interval variable dose (VIVD) scheme. However, the resulted schedules seemed problematic in for both patient's side and clinician's side due to its randomness. Therefore, in next two cases, dose days were decided with optimum fixed-valued interval. These two schemes are more feasible in times when the dose taking must be completely monitored by clinicians/hospital staffs [HARROLD, 2005]. When FIVD is formulated, periodicity has not been imposed rather near periodicity is achieved from the $4^{\text {th }}$ week in the optimal result (negligible variation of dose levels). So for more simplification and clinical relevance, in our final step we have approached periodicity and proposed periodic dose pattern.

In real case scenario, majority of the model parameters are related to physiological conditions of patients, internal drug absorption/metabolism, drugs to be used in treatments, state/position of tumor when it was detected etc. The model parameters can vary in every case and also these vary in-vivo or insilico experiments conducted under environment but at different instances. So to develop more accurate/realistic models for specific cancer, existing/commonly used parameters are modified/adjusted with empirical data or new parameters are added to existing model.

\section{Conclusion and Discussion}

Chemotherapy schedules recommended by oncologists/clinicians are obtained from experiential verification with preclinical and clinical trials [HARROLD, 2005] as well as theoretical considerations. Engineers have dealt with the problem as a process to control and tried to offer more and more optimal drug schedules. Yet, there is gap between engineering tactics and clinical implementation. In this investigation, a design method of optimal cancer chemotherapy schedules using GA is portrayed that focuses to bridge this gap.

The work emphasizes more on clinical relevance and patient's compared to mathematical optimality. The discontinuity in schedules offer treatment holidays to patient's to heal the damages due to chemotherapy. FIVD and periodic dose schemes are highlighted in this work. Their preference, when direct observation of hospital staffs is obligatory, is also projected. However, to truly make an impact, all of the designing work discussed above will require clinical testing in collaboration with oncologists and efforts are underway with that incentive.

This kind of discontinuous and periodic treatment scheme can be incorporated with periodic evaluation of treatment responses (e.g. 
tumor size, toxicity etc.) and modification of the current schedule accordingly in a closed loop environment. Furthermore, the same design method can be extended in planning multi-drug or combination chemotherapy regimen. 


\section{References}

[ADAMS, R.A. et al. 2011]

[ALAM, N. et al. 2013a]

[ALGOUL, S. et al. 2010]

[ALGOUL, S. et al. 2011]

[ALAM, MS. et al. 2013]

[AL-MAMUN, MA et al. 2012]

[AL-MAMUN, MA et al. 2013]

[CLARE, S.E. et al. 2000]

[DEB , K. 2008]

[HARROLD, J.M. 2005]

[HOLLAND, J.H. 1975]

[KIRAN, K. et al. 2008]

[KAUFMAN, D.C. et al . 2001]

[KAZMI, N. et al. 2012a]
ADAMS, R.A., MEADE A.M., SEYMOUR, M.T. et al. Intermittent versus continuous oxaliplatin and fluoropyrimidine combination chemotherapy for first-line treatment of advanced colorectal cancer: results of the randomized phase 3 MRC COIN trial.

Lancet. Oncol., 12, pp-642-653, 2011.

ALAM, N., SULTANA, M., ALAM, M.S., et al. Chemotherapy Dose Schedule Optimization Using Genetic Algorithm.

Distributed Computing and Artificial Intelligence, Advances in Intelligent Systems and Computing, pp-503-511, Springer International Publishing, 2013.

ALGOUL, S., ALAM, M.S., HOSSAIN, M.A. et al. Multi-objective Optimal Chemotherapy Control Model for Cancer Treatment.

Springer Journal on MBEC, 49(1), pp-51-65, 2010. Springer-Verlag Berlin.

ALGOUL, S., ALAM, M.S., SAKIB, K. et al. MOGA-based Multi-drug Optimization for Cancer Chemotherapy.

AISC, 93, pp-133-140, 2011. Springer-Verlag Heidelberg.

Alam, M.S., Hossain M.A., Algoul, S., et al. Multi-Objective Multi-drug Scheduling Schemes for Cell Cycle Specific Cancer Treatment.

International Journal of Computers \& Chemical Engineering, 58, pp-1432, 2013.

Al-Mamun M.A., Kazmi, N., Hossain, M. A., Vickers, P., and Jiang, Y., An intelligent decision support system for personalized cancer treatment. In: CIS2012: 11th IEEE Conference on Cybernetic Intelligent Systems, 2324 August 2012, Limerick, Ireland

Al-Mamun, M.A., Hossain, M.A., Alam, M.S., Bass, R., A Cellular automaton model of the effects of maspin on cell migration. Advances in Intelligent Systems and Computing Volume, 222, pp 53-60.

CLARE S.E., NAKHLIS F., PANETTA J.C. Molecular biology of breast cancer metastasis: The use of mathematical models to determine relapse and to predict response to chemotherapy in breast cancer. Breast Cancer Res. 2 (6), 2000, pp-430-435.

$\mathrm{DEB}, \mathrm{K}$. Introduction to evolutionary multiobjective optimization.

Multiobjective Optimization, pp. 59-96, 2008. Springer-Verlag Berlin Heidelberg

HARROLD, J.M. Model-based design of cancer chemotherapy treatment schedules.

Ph.D. Thesis, 2005, University of Pittsburgh, USA.

HOLLAND, J.H. Adaptation in natural and artificial system.

University of Michigan Press, 1975. USA

KIRAN, K., JAYACHANDRAN, D., LAKSHMINARAYANAN, S. Multi-objective Optimization of Cancer Immuno-Chemotherapy.

In Proc. ICBME, Springer, pp-1337-1340, 2008.

KAUFMAN, D.C., CHABNER, B.A. Clinical strategies for cancer treatment: the role of drug.

In Chabner, B.A., Longo, D. L. (eds.). Cancer Chemotherapy and Biotherapy: Principles and Practice, Lippincott, Williams and Wilkins, pp1-16, 2001/

Kazmi, N., Hossain, MA., Phillips, RM., Al-Mamun, MA., Bass, R.,. Avascular tumour growth dynamics and the constraints of protein binding

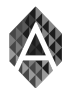

Advances in Distributed Computing And Artificial Intelligence Jornual 
[KAZMI, N. et al. 2012b]

[MARTIN, R. et al. 1990]

[MARTIN, R. et al. 1994]

[MCCALL, J. et al.,1999]

[MCCALL, J. et al.,2001]

[MCCALL, J. et al., 2004]

[MCCALL, J. et al. 2008]

[NORTON, L. 1988]

[SLINGERLAND, J.M. et al. 1998]

[SWAN, G.W. 1986]

[THURSTON, D.E. 2006]

[URQUHART, J. et al. 1998] for drug transportation. J Theor Biol. Nov 21;313:142-52.

Kazmi, N., Hossain, MA., Phillips, RM., A hybrid cellular automaton model of solid tumor growth and bioreductive drug transport. IEEE/ACM Trans Comput Biol Bioinform. Nov-Dec;9(6):1595-606

MARTIN, R. B., FISHER, M. E, MINCHIN, R. F., TEO, K. L. (1990). A mathematical model of cancer chemotherapy with an optimal selection of parameters.

Math. Biosci., 99, pp-205-230.

MARTIN, R, TEO, K.L. Optimal Control of Drug Administration in Chemotherapy Tumor Growth.

World Scientific, 1994. River Edge, NJ, USA.

MCCALL, J., PETROVSKI, A. A decision support system for cancer chemotherapy using genetic algorithms.

In Proceedings of the international conference on computational intelligence for modeling, control and automation, pp-65-70, 1999.

MCCALL, J., PETROVSKI, A. Multi-objective optimization of cancer chemotherapy using evolutionary algorithms.

Evolutionary Multi-Criterion Optimization, Lecture Notes in Computer Science, 1993, pp-531-545, 2001, Springer-Verlag Berlin Heidelberg.

MCCALL, J., PETROVSKI, A. SUDHA B. Optimizing cancer chemotherapy using particle swarm optimization and genetic algorithms. In Proceedings of the 8th international conference on parallel problem solving from nature, Lecture notes in computer science. Springer, Berlin, 3242, pp-633-641, 2004.

MCCALL, J., PETROVSKI, A., SHAKYA, A. Evolutionary algorithms for cancer chemotherapy optimization.

In Fogel, G.B., Corne, D.W., Pan Y. (eds.). Computational Intelligence in Bioinformatics, John Wiley \& Sons, Inc., pp-265-296, 2008. Hoboken, NJ, USA.

NORTON, L. A Gompertzian model of human breast cancer growth.

Cancer Res., 48, pp-7067- 7071.

SLINGERLAND, J.M., TANNOCK, I.F. Cell Proliferation and Cell Death.

The Basic Science of Oncology, McGraw-Hill, 1998, pp-134-165.

SWAN, G. W. Cancer chemotherapy: Optimal control using the VerhulstPearl equation.

Bull. Math. Biol., 48(3/4), pp-381-404, 1986.

THURSTON, D.E. Chemistry and pharmacology of anticancer drugs.

CRC Press, 2006. Boca Raton, Florida, USA

URQUHART J., KLERK E.D. Contending paradigms for the interpretation of data on patient compliance with therapeutic drug regimens.

Stat. Med., 17, 1998, pp-251-267. 